\title{
Mobile-Robot Navigation through Fuzzy Behavioral Algorithm \& Vector-Polar Histogram Algorithm
}

\author{
Alaudeen Basha ${ }^{1}$, V. Vijayakumar ${ }^{2}$ \\ ${ }^{1}$ Department of Information Technology, AMET University, Chennai, India \\ ${ }^{2}$ School of Computer science \& Engineering, VIT University, Chennai, India
}

\begin{tabular}{l}
\hline Article Info \\
\hline Article history: \\
Received Nov 21, 2017 \\
Revised Jan 29, 2018 \\
Accepted Feb 17, 2018 \\
\hline
\end{tabular}

\section{Keywords:}

Behaviour-based Control

Fuzzy Control

Robot Navigation

Vector Polar

\begin{abstract}
The route of self-governing ground vehicles through general conditions has gotten generous research consideration. Be that as it may, the writing contains not very many correlations of the course ideal models for AGVs, particularly for calculations utilising range discoverers. The fluffy behavioural approach and vector field histogram (VFH) approach are outstanding strategies that can be actualised using range discoverers. This paper will concentrate on looking at their structure, simplicity of programming and calculation tuning, and execution. Both methodologies actualised on a Pioneer 2 robot, outfitted with a SICK laser run discoverer.
\end{abstract}

Copyright $@ 2018$ Institute of Advanced Engineering and Science. All rights reserved.

\section{Corresponding Author:}

Alaudeen Basha,

Department of Information Technology,

AMET University,

Chennai, India.

\section{INTRODUCTION}

Significant research has been committed to exploring self-ruling ground vehicles through dubious conditions. Receptive frameworks that utilisation nearby guide data regularly decided for this assignment as a result of their computational proficiency. The fluffy behavioural approach and the vector field histogram approach are two essential receptive methodologies. Be that as it may, the writing contains not very many examinations of these two procedures. Thus, this paper gives an analysis of their structure, simplicity of programming and tuning, and execution. The particular fluffy behavioural approach considered here was first created in [1]-[4], and prompted the calculation of [5], [6] for the route in greatly jumbled conditions, which utilised as a part of the correlation of this paper. In this calculation, the practices are composed using inclination based voting and summon combination. As needs are, the count will be known as the preferencebased fluffy behavioural calculation. Comparative estimates for various applications are given in [7], [8]. The VFH approach initially created. The underlying methodology utilised sonars as the range discoverers. Because of the mistake of sonar estimations, the calculation remunerates by considering the sureness of the presence of the deterrents. This approach was altered to utilise a laser run discoverer and brought about the vector polar histogram calculation, which employed as a part of this paper. In this technique, the sensor readings are utilised to construct a polar histogram, which is additionally sifted and decoded to get the controlling course. Extra applications using this calculation illustrated. This paper introduces a quantitative and subjective examination between the PBFB calculation and the VPN calculation, in light of algorithmic structure, the simplicity of programming, tuning multifaceted nature, and execution. The correlation just considers low-speed cases since both strategies are not ready to successfully find vehicle progression [11] presents a new algorithm used by to pursue littered trash based on the combination of cell decomposition algorithm and fuzzy. A fuzzy algorithm typically used for path planning which serves to detect an obstacle by 
using sensors it can steer robots towards the goal position. In [12] it presents the work, the model of the DC motor incorporated within a larger simulation of a PMSG system with the DC motor acting as the prime mover. In [13] the simulation results show that the problem of chattering inhibited naturally, and the ability of anti-interference, anti-parameters perturbation, stability and control quality of the system are improved effectively. At the same time, the scheme is straightforward and suitable for engineering application. In paper [14], presented an energy-efficient and reliable routing protocol for mobile WSNs. The protocol E2R2 is hierarchical and cluster-based. Each cluster contains one $\mathrm{CH}$ node and the $\mathrm{CH}$ node assisted by two DCH nodes, which are also called cluster management nodes.

\section{DESCRIPTION OF THE PBFB AND VPN ALGORITHMS}

This section describes the PBFB and VPN algorithms.Additional details can be found in [9], [10].

\subsection{PBFB Algorithm}

A behavioural framework comprises of a limited arrangement of parallel running practices, and a charge combination unit. Heuristic Approach to Supervised Learning for Intrusion Detection [10] presented the education system. An arrangement of option control charges is kept by this order combination unit and is likewise known by every conduct. In an inclination based behavioural framework, the practices react to their particular boosts by communicating their preference level to each of the summon choices. The inclinations melded by the summon combination unit to acquire the course of movement that best fulfils the different practices. As shown in Figure 1 the methods utilised as a part of the PBFB calculation are front snag shirking, left obstruction evasion, right deterrent shirking, and objective chasing. The summon choices are large left turn, slight left turn, no turn, slight right turn, and high right turn. Every conduct is a fluffy framework, and the inclinations communicated as fuzzy sets. An inherent disadvantage of current behaviour structures is the absence of coherence in the charges, i.e., each order is permitted just to have a discrete esteem and middle of the road regards rejected. Fluffy practices inalienably wipe out this issue. The yield bearing can be any point inside 1800 , which gives smooth movement.

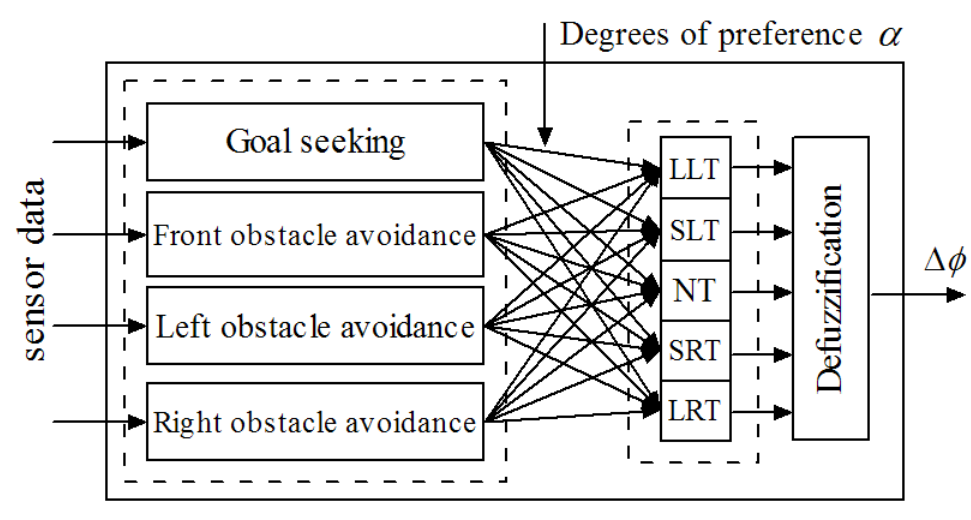

Figure 1. Definite preference based Behaviour control system

An individual element of the PBFB calculation is the sensor design. As appeared in Figure 2, the sensor readings are gathered into nine parts, marked $\mathrm{C} 1, \mathrm{C} 2, \mathrm{C}$. The small sensor readings in every segment are new contributions to the fluffy framework for every conduct: $\mathrm{C} 1$ to $\mathrm{C} 3$ for the left divider following conduct, C4 to C6 for the impediment shirking behaviour, and C7 to C9 for the correct divider the following manner. A unique favourable position of this sensor setup is that the robot can make full utilisation of the sensor examining information without expanding the contributions to the fluffy frameworks, which could significantly build the computational cost. 


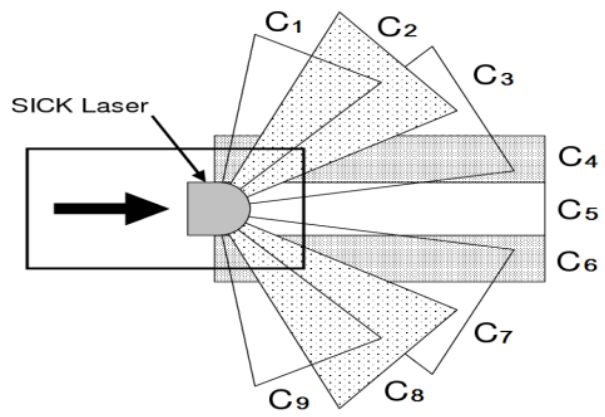

Figure 2. Sensor Configuration of PBFB Algorithm

\subsection{VPH Algorithm}

The possibility of the VPH calculation is like the first VFH calculation aside from that the VPH calculation utilises a laser as the range sensor rather than sonar. As appeared in Figure 3, the sensor information mapped into a polar separation dissemination, where $r$ is the separation of the robot to the deterrent at the examining edge $\varphi$. This hindrance appropriation is additionally changed into a polar vector histogram as appeared in Figure 4, where they pivot speaks to the separation, the $\mathrm{x}$ hub is the comparing edge, and $\mathrm{T}$ is the edge. The competitors found by applying the edge $\mathrm{T}$ to the polar vector histogram. Finally, a precisely picked cost work assesses the hopefuls keeping in mind the end goal to get the most attractive guiding heading. Figure 5 demonstrates the schematic graph for the VPH calculation.

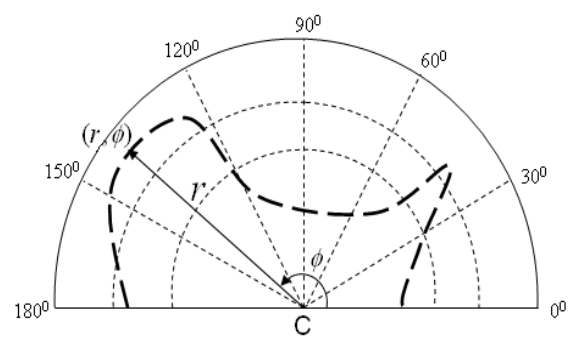

Figure 3. Obstacle polar disturbance distribution

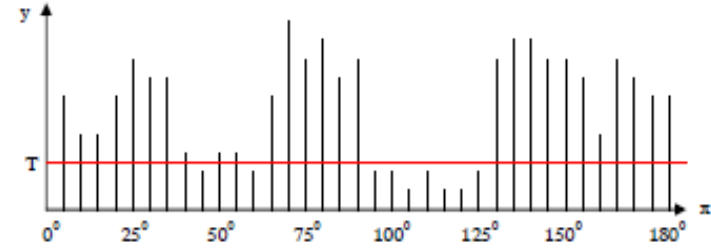

Figure 4. Vector Polar Histogram

\section{COMPARISON OF THE PBFB AND VPH ALGORITHMS}

This section provides a comparison of the PBFB and VPHalgorithms based on their first structure ease of programming and tuning and experimental performance.

\subsection{Fundamental Structure}

Both the PBFB and VPH calculations are responsive. Subsequently, just nearby guide data utilised as a part of the calculation. Therefore, the created way has no assurances of global optimality and breaking point cycle conduct can happen with either math. By and by, these calculations have been exhibited to give important route in complex and unstructured conditions regularly. Confine cycle, or nearby minima can abstain from utilising an assortment of methodologies. The PBFB calculation, has a parallel structure that enables each behavioural sub-framework to at the same time figure; this empowers the utilisation of various processors to accomplish computational productivity. The VPH calculation utilises serial processing; in any case, the calculation is not tedious because of the straightforwardness of the math. As appeared in Figure 5, the VPH calculation utilises an immediate technique to display the hindrance appropriation. The power of this calculation mainly originates from the high exactness of the laser sensor estimations. Interestingly, in the PBFB calculation the range information is grouped into three fuzzy sets: short, medium, and long. Subsequently, the PBFB calculation is uncaring to mistakes in the sensor readings. At the point when high exactness sensors are not accessible, the more mind-boggling VFH calculation must be utilised rather than the VPH calculation, while the PBFB calculation ought to encounter negligible execution debasement. 


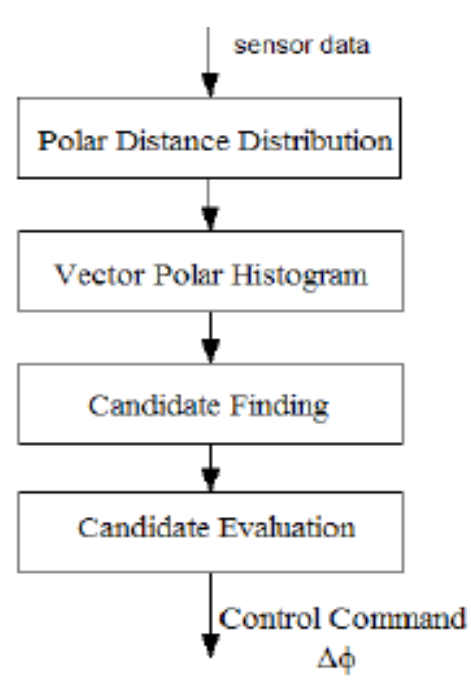

Figure 5. Schematic of VPH approach

\subsection{Ease of Programming and Tuning}

In the VPH method, an essential part is to decide a smooth and precise vector polar histogram, which was a great deal more entangled in the first VFH approach given the utilisation of less exact sonar. Due to the usage of the laser, the programming of the VPH method is exceedingly streamlined. The tuning factors are the edges that bar the undesirable bearings comparing to the nearby hindrances from the polar vector histogram, the division numbers for full and restricted openings from the polar vector histogram, and the parameters used to characterise the cost work. These qualities are usually effectively dictated by the measure of the robot, the impediment thickness, and the objective position. Interestingly, the PBFB calculation requires more push to program and tune. To start, it has four arrangements of fluffy frameworks, each of which is controlled by the comparing practices. Second, for smooth and efficient movement, the fuzzy guidelines of each fluffy framework should be tried by experimentation. Once in a while, even the fuzzy sets, including the decision of the comparing participation capacities might be tuned. At long last, as examined in the writing [1], [7], it is tough to find appropriate coordination instrument and a defuzzification strategy that can proficiently meld the fluffy behavioural frameworks and interpret the alluring course. The PBFB tackles this issue by utilising an inclination based structure and the focal point of the biggest range for defuzzification.

\section{EXPERIMENTAL RESULTS}

As a measure of the execution of the proposed technique, the trial comes about acquired utilising both the PBFB, and VPH calculations looked. The accompanying ten tests used as a reason for examination. In the trials, a Pioneer 2 robot, furnished with a SICK LMS 200 laser, was utilised for both calculations. Figure 6 gives a depiction of these tests, which directed in an indoor region of around $3.6 \mathrm{~m} \times 6.0 \mathrm{~m}$. The average separation of the two nearest snags is around 1.4 times the width of the robot $(\approx 50 \mathrm{~cm})$ in each of the "woodland" scenes.

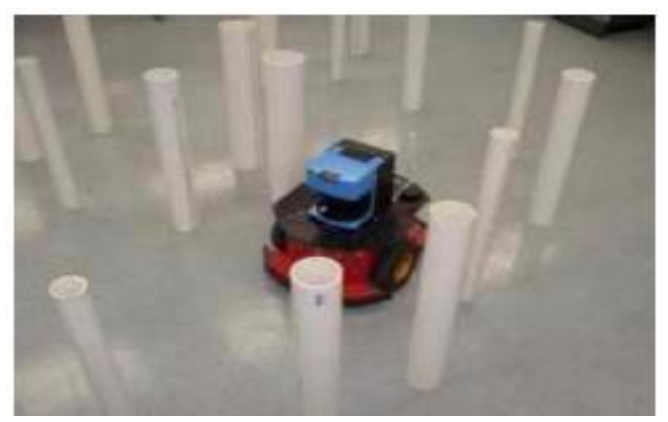

Figure 6. Robot Navigating through Dense Obstacle Field 
Situation 1, appeared in Figure 7, first requires the robot to go through a thin "hall." When the robot draws near to the objective, a mass of impediments shields the robot from going straight to the objective. Effect of Lignite Fly Ash and Composted Coir Pith on Cultivable Soils [9] described the effects of the lignite fly ash. The robot takes after the divider despite the fact that this obliges it to move far from the objective incidentally. Finally, the robot stays away from the divider to achieve the goal. The two calculations deliver fundamentally the same as ways because the way in this situation is to a large degree dictated by the deterrent arrangement. Case 2, appeared in Figure 8, speaks to more difficult circumstances that outline the capacity to explore little holes and even move in the opposite direction of the objective when important to keep away from deterrents. The robot can even dismiss generously from the purpose in the wake of drawing near to it, the length of it finds no safe way specifically to the objective. Once more, both calculations created comparable ways because of the confinements of the hindrance setup.

Situations 3 through 7, appeared in Figures 9 through 13, indicate thick woodlands situations that have various ways to the objective. The robot needs to pick an ideal approach to go through these conditions. The calculations pick on a fundamental level distinctive ways in each of these situations.

Situations 8, appeared in Figure 14, speaks to a circumstance that delineates the capacity to take after a mass of obstructions, then find and go through an "entryway." Both calculations take after comparable ways.

Situation 9, appeared in Figure 15, speaks to a snag design that enables the robot to pick distinctive bearings at the earliest reference point of the way. The VPH strategy empowers the robot to start its way by making a beeline for the objective notwithstanding when the neighbourhood world toward the goal is moderately jumbled yet acceptable. The behavioural technique picks a heading far from the target and takes after away with smoother movement.

Situation 10, appeared in Figure 16, speaks to a circumstance where the target is lined up with the robot's underlying heading. In any case, the objective of shows a capacity to dodge the deterrents and discover a way before it to achieve the goal. Again the calculations pick two distinct ways.

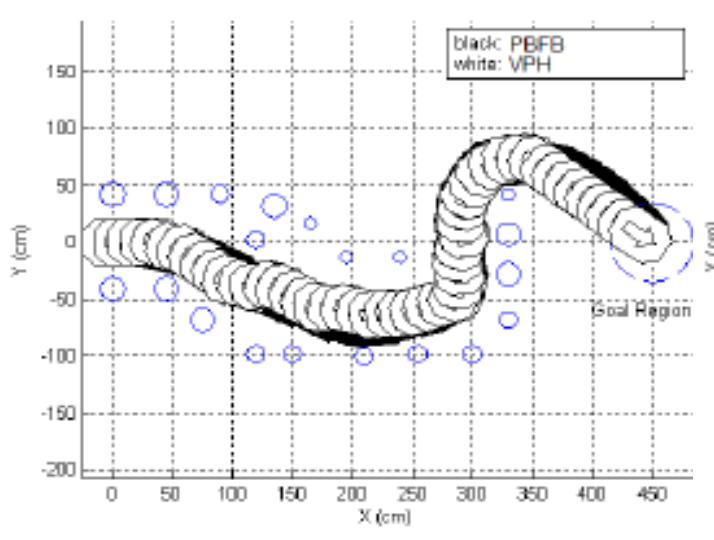

Figure 7. Scenario 1

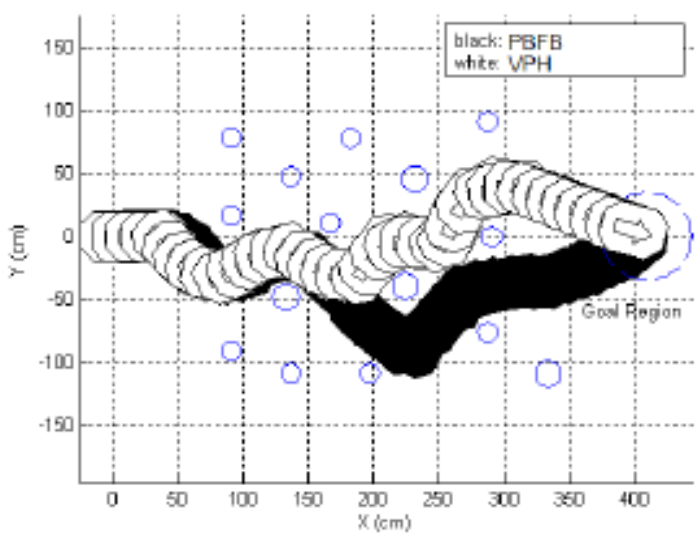

Figure 9. Scenario 3

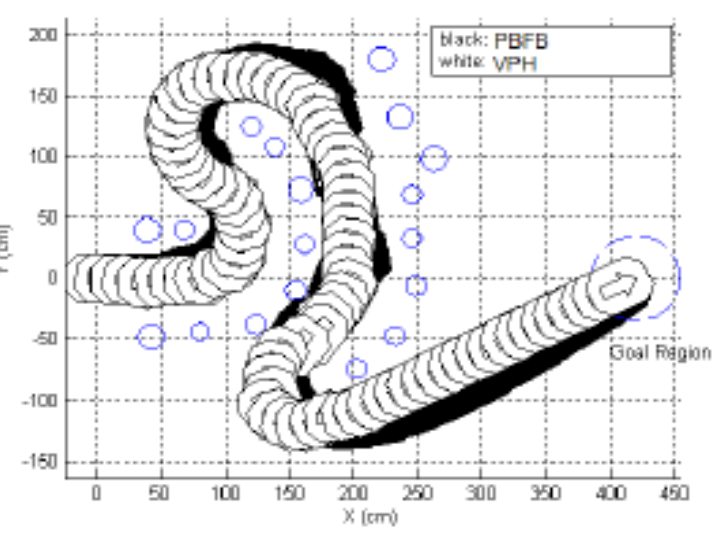

Figure 8. Scenario 2

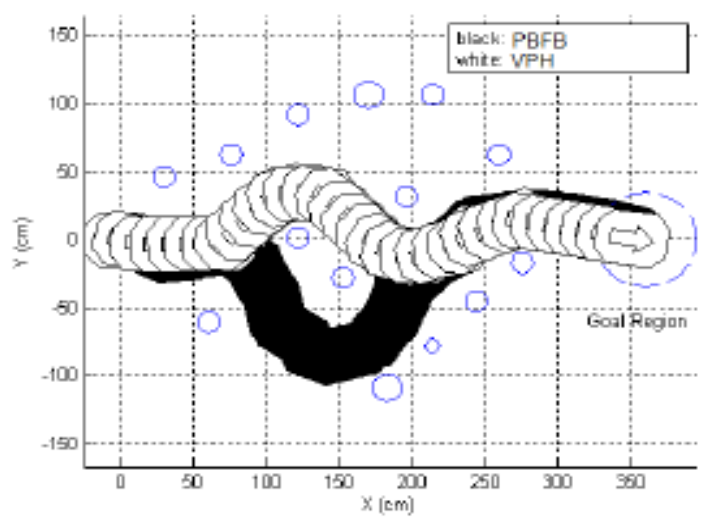

Figure 10. Scenario 4 


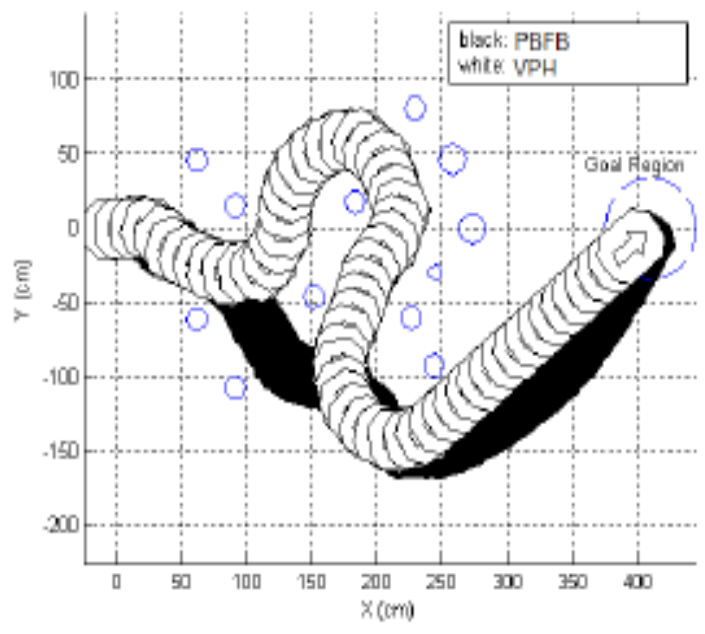

Figure 11. Scenario 5

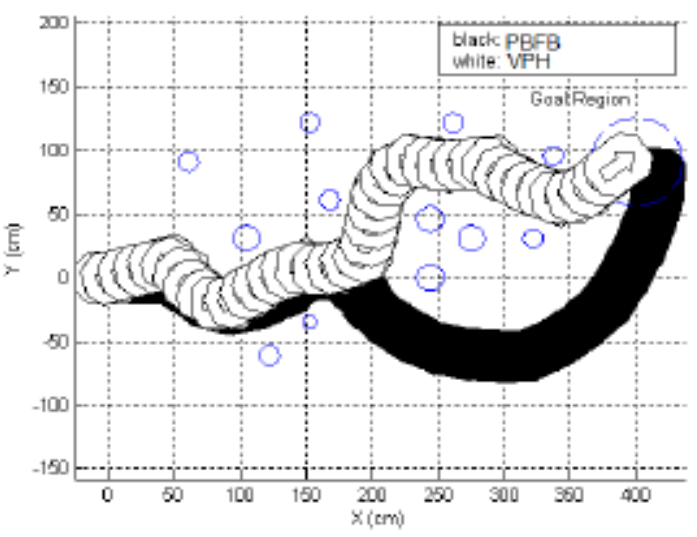

Figure 13. Scenario 7

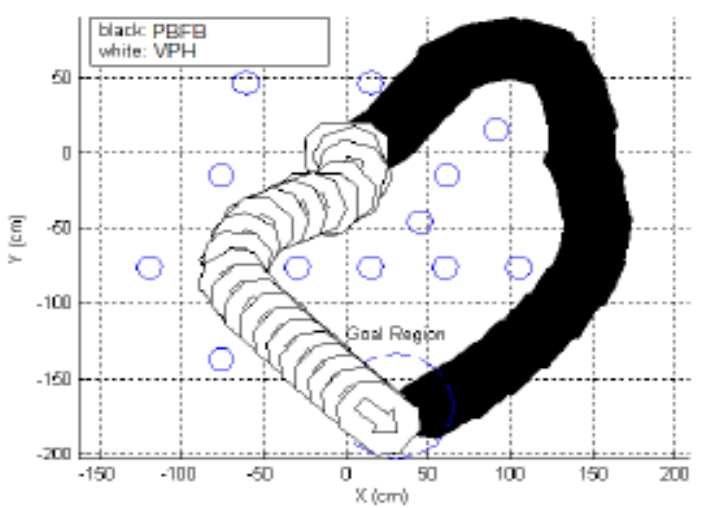

Figure 15. Scenario 9

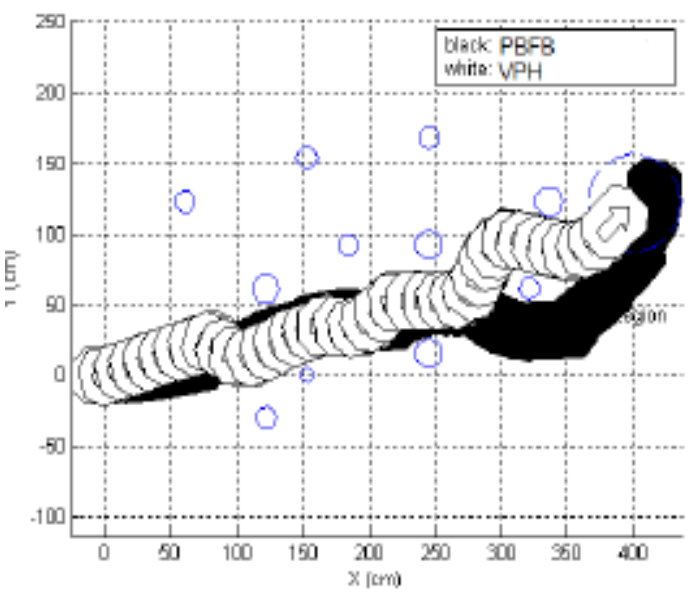

Figure 12. Scenario 6

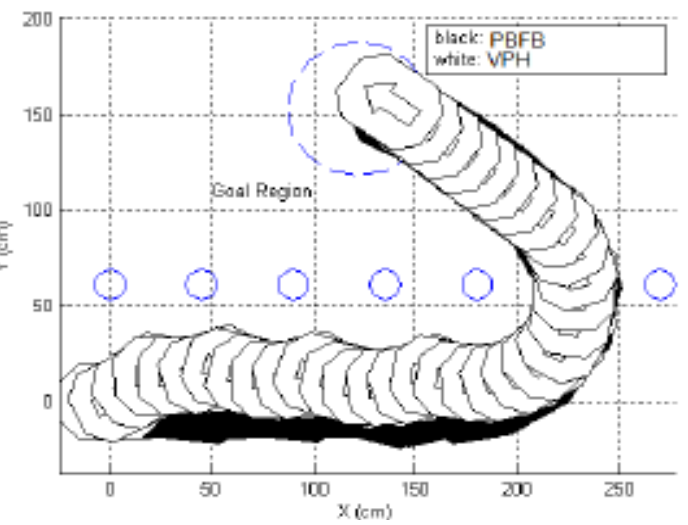

Figure 14. Scenario 8

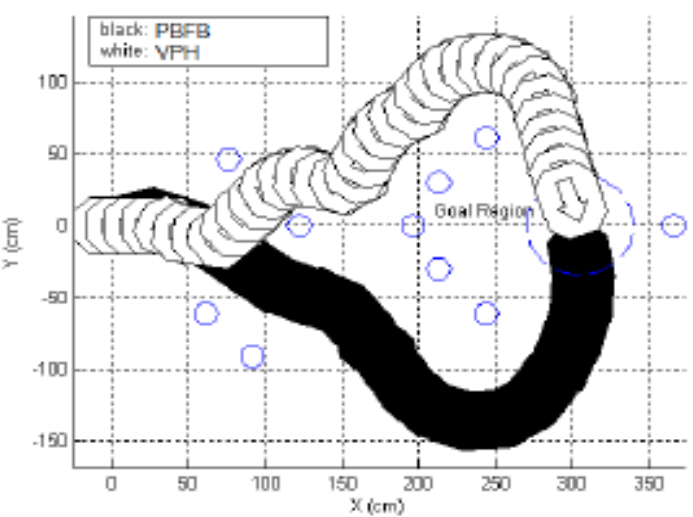

Figure16. Scenario 10

\section{CONCLUSION}

This paper introduces a subjective and quantitative correlation between the inclinations based fluffy behavioural calculation and the vector polar histogram calculation given their structures, simplicity of programming, calculation tuning, and their execution in the different timberland like situations. From the perspective of fundamental multifaceted nature and simplicity of programming and tuning, the VPH 
approach takes less programming and implementation time. Subsequently, the VPH is the better decision for undertakings where execution speed is exceedingly imperative, for instance in a lab that is a piece of an application for autonomy course. The PBFB calculation, as a rule, produces much smoother ways, demonstrating that the PBFB calculation would be the better decision for assignments where vitality utilisation and movement solace are essential, for instance, in the outline of semi-self-ruling wheelchairs for the disabled.

\section{REFERENCE}

[1] Payton D W, Rosenblatt J K, Keirsey D M. Plan guided reaction. IEEE Transactions on Systems, Man, and Cybernetics. 1990; 20(6); 1370-1382.

[2] Yen J, Pfluger N. A fuzzy logic based extension to Payton and Rosenblatt's command fusion method for mobile robot navigation. IEEE Transactions on Systems, Man, and Cybernetics. 1995; 25(6); 971-978.

[3] Saffiotti A, Ruspini E H, Konolige K. Blending reactivity and goal-directedness in a fuzzy controller. In Fuzzy Systems, Second IEEE International Conference. 1993; 134-139.

[4] Saffiotti A, Konolige K, Ruspini E H. A multivalued logic approach to integrating planning and control. Artificial intelligence. 1995; 76(1-2); 481-526.

[5] Selekwa M F, Dunlap DD, Collins E G Implementation of multi-valued fuzzy behaviour control for robot navigation in cluttered environments. In Robotics and Automation, 2005. ICRA 2005. Proceedings of the 2005 IEEE International Conference. 2005, pp. 3688-3695.

[6] Dunlap D D. Implementation of multi-valued fuzzy behaviour control for navigation in cluttered environments. 2004.

[7] Safiotti A. Fuzzy logic in autonomous robotics: behaviour coordination. In Fuzzy Systems, 1997. Proceedings of the Sixth IEEE International Conference. 1997; 1; 573-578.

[8] Watanabe K, Izumi K, Maki J, Fujimoto K. A fuzzy behaviour-based control for mobile robots using adaptive fusion units. Journal of Intelligent \& Robotic Systems. 2005;42(1); 27-49.

[9] Elavalagan V A. Effect of Lignite Fly Ash and Composted Coir Pith on Cultivable Soils

[10] Shanthi H J, Anita E M. Heuristic Approach of Supervised Learning for Intrusion Detection. Indian Journal of Science and Technology. 2014; 7(S6); 11-14.

[11] Tatiya Padang Tunggal, Andi Supriyanto, Nur Mukhammad Zaidatur Rochman, Ibnu Faishal, Imam Pambudi and Iswanto Iswanto. Pursuit Algorithm for Robot Trash Can Be Based on Fuzzy-Cell Decomposition, International Journal of Electrical and Computer Engineering (IJECE), Vol 6, No 6, December 2016, pp. 2863-2869.

[12] Bouzid Mohamed Amine, Zine Souhila, Allaoui Tayeb and Massoum Ahmed. Adaptive Fuzzy Logic Control of Wind Turbine Emulator, International Journal of Power Electronics and Drive Systems (IJPEDS), Vol 4, No 2, June 2014, pp. 241-255.

[13] Fenglan Jia, Li Hou, Yongqiao Wei, Yunxia You and Lili Yan, Adaptive Fuzzy Sliding Mode Control for Hydraulic Servo System of Parallel Robot, Indonesian Journal of Electrical Engineering and Computer Science, Vol 12, No 6, June 2014, pp. 4125-4133.

[14] Rajesh. D, M. Firoja Banu, D. Stella and Ansila. P. Grace. CH Panel Based Routing Scheme for Mobile Wireless Sensor Network, International Journal of MC Square Scientific Research (IJMSR), Vol 8, No 1, 2016, pp. 183-198. 